\title{
New features in MEK retinopathy
}

\author{
Pallavi Tyagi and Cynthia Santiago
}

\begin{abstract}
Background: The use of molecularly targeted therapy is becoming widespread in oncology. These agents cause tumour-specific genetic alterations in signal transduction pathways, hence less generalised toxicity. Dabrafenib, a BRAF inhibitor and Trametinib, a MEK inhibitor are two molecularly targeted agents recently approved for treatment of advanced, unresectable melanomas. MEK retinopathy is a recently introduced term describing retinal toxicity secondary to MEK inhibitors.

Case presentation: A 71-year-old man presented with 'circular, green patches' in his central vision for 2 weeks. He had multiple relapsed stage IV BRAF gene mutant malignant melanoma. He was on treatment with Dabrafenib (Tafinlar) for 7 months and Trametinib (Mekinist) for 4 months respectively. The fundus looked normal. The OCT scan showed bilateral symmetrical cystoid macular edema, intraretinal and subretinal fluid, thickening of elliposoid zone and subretinal granular deposits. The symptoms resolved with temporary cessation of chemotherapy but OCT signs persisted.

Conclusion: This case report identifies two new remarkable features of MEK retinopathy as thickening of ellipsoid zone and 'starry sky' pattern of distribution of subretinal granular deposits. These changes signify photoreceptors/ RPE toxicity and dysfunction. The subretinal granular deposits showed increased autofluorescence suggested abnormal lipofuscin clearance due to RPE dysfunction. The molecularly targeted therapy has revolutionized the cancer treatment and increased the survival rate. These agents are relatively new and recently approved for clinical use and most of them are associated with ocular toxicities. Awareness of ocular symptoms, side-effect profile of drugs, monitoring regime and liaison between oncologist and eye care professional with ocular imaging is key to early diagnosis and management of ocular adverse events.
\end{abstract}

Keywords: MEK retinopathy, Molecular chemotherapy, MEK inhibitors, BRAF inhibitors, Ocular toxicity

\section{Background}

Anticancer therapy as chemotherapeutic agents, hormonal and molecular targeted treatments can produce ocular toxicity. The ocular adverse events (AE) can occur due to effect on cellular proliferation, disruption of ocular immune privilege or direct toxicity to ocular structures. Whereas conventional chemotherapy targets both normal and rapidly dividing cells, newer agents tend to exploit tumour-specific genetic alterations in signal transduction pathways.

Almost $50 \%$ of malignant melanomas have somatic mutation of BRAF gene [1]. BRAF inhibitors act on enzyme B-Raf, which plays a role in the regulation of cell growth. Dabrafenib is a BRAF inhibitor that was approved by National Institute of Clinical Excellance (NICE) in United

\footnotetext{
* Correspondence: palv2007@gmail.com

Department of Ophthalmology, Aberdeen Royal Infirmary, Foresterhill Road Aberdeen, AB25 2ZN, UK
}

(C) The Author(s). 2018 Open Access This article is distributed under the terms of the Creative Commons Attribution 4.0 International License (http://creativecommons.org/licenses/by/4.0/), which permits unrestricted use, distribution, and reproduction in any medium, provided you give appropriate credit to the original author(s) and the source, provide a link to the Creative Commons license, and indicate if changes were made. The Creative Commons Public Domain Dedication waiver (http://creativecommons.org/publicdomain/zero/1.0/) applies to the data made available in this article, unless otherwise stated. BRAF V600 mutation-positive melanoma in 2014. Another molecular pathway commonly affected in malignant melanomas is mitogen-activated protein kinase/ extracellular regulated kinase (MAPK/ERK) also known as MEK pathway. Trametinib is a MEK inhibitor that inhibits MEK1 and MEK2 genes. Clinical trial data demonstrated that resistance to Dabrafinib and other BRAF inhibitors occurs within 6 to 7 months [2]. This resistance can be overcome by the combination of BRAF inhibitor Dabrafenib with the MEK inhibitor Trametinib [2-4]. Trametinib in combination with Dabrafenib for treating unresectable or metastatic melanoma was approved by NICE in UK in 2016.

Whereas Dabrafenib disturbs ocular immune privilege and is commonly associated with uveitis, Trametinib and other MEK inhibitors usually develop retinal toxicity considered as 'class effect' $[5,6]$. 'MEK retinopathy' is an umbrella term used to describe the dose- and time- 
dependent retinal side-effects observed with MEK inhibitor therapy $[5,6]$. MEK inhibitor clinical trials have reported ocular toxicities in $5-38 \%$ of treated patients. [7]. The wide range of incidence may be due to the lack of uniformity in the description, diagnosis and reporting of the same condition, differences in potency of MEK inhibition, schedule of administration and the frequency of ophthalmologic assessment across trials [5].

\section{Case presentation}

A 71-year-old presented to community optometrist with 'patchy vision' and 'green circular patches' in central vision of both eyes for 2-3 weeks. He was noted to have bilateral cystoid macular edema on optical coherence tomography (OCT) scan and was referred to hospital eye services (Fig. 1). The patient had previously received annual ophthalmic assessments and retinal imaging by optometrist which were unremarkable. He had been seen in eye clinic with left sided herpes zoster ophthalmicus disciform keratitis and uveitis 7 months previously with no retinal involvement. His medical history included hypertension and multiple relapsed stage IV BRAF gene mutant malignant melanoma involving skin, liver and spleen for 11 years. The melanoma nodules were present on buttock, back and periumblical region. He had been treated with surgical excision of nodules, inguinal nodal and femoral vein resection and radiotherapy with consequent lymphoedema of lower limb over years. His most recent melanoma relapse was 15 months previously and was treated with 3 cycles of Ipilimumab which were later discontinued due to uncontrolled diarrhoea. Subsequently, he was found to have enlargement of periumbilical and liver melanoma nodules with appearance of a new nodule in the lung. At this point he was commenced on molecularly targeted oral chemotherapeutic agents, Dabrafenib (Tafinlar) $150 \mathrm{mg}$ twice a day (BD) initially followed by Trametinib (Mekinist) $2 \mathrm{mg}$ once a day (OD) for 7 months and 4 months respectively. Since the molecularly targeted therapy is known to have ocular side-effects the patient was given comprehensive side-effect profile information at the initiation of treatment and was advised to seek immediate ophthalmic assessment if any visual disturbance. This prompted him to see his optometrist at the onset of symptoms. In view of the ocular symptoms, the oncology team advised him to stop his chemotherapeutic drugs, Dabrafenib and Trametinib and requested specialist ophthalmology review. He was seen in eye clinic 5 days after stopping treatment. His symptoms were marginally better. The visual acuity was $6 / 6$ in both eyes on Snellen's chart. The anterior segments were normal. Fundus examination showed healthy optic discs, macula showed dull foveal reflex with normal periphery and retinal vasculature (Fig. 2). The OCT scan showed intra-retinal and sub-retinal fluid on macula with cystoid changes in the peri-foveal area. More strikingly, there was significant thickening of the ellipsoid zone and sub-retinal granular deposits overlying an intact looking retinal pigment epithelium (RPE). Infrared reflectance image (IRR) showed multiple hyper-reflective lesions that corresponded to the sub-retinal granular deposits on OCT imaging. These deposits were distributed in bilateral symmetrical 'starry sky' appearance on the macula (Fig. 3). The choroid appeared unaffected with choroidal thickness of $180 \mu \mathrm{m}$ in right eye and $225 \mu \mathrm{m}$ in left eye. On autofluorescence (AF) imaging, the macular sub-retinal granular lesions showed increased autofluorescence (Fig. 4). The macular edema looked improved compared to scans sent by community optometrist.

His chemotherapeutic drugs, Dabrafenib and Trametinib were withheld for 3 weeks. He noticed subjective improvement in symptoms with clearing of dark patches in his vision within 10 days. His vision, fundus and OCT appearances were unchanged. He was recommenced on reduced dose of Dabrafinib (100 mg) BD and Trametinib (1 mg) OD after 3 weeks to prevent melanoma relapse. $\mathrm{He}$ remained visually asymptomatic and clinically unchanged on this dose for 6 months. Over time, the cystoid macular edema resolved in the perifoveal region with reduction in the intra-retinal and sub-retinal fluid. The ellipsoid zone thickening and subretinal deposits remained unchanged (Fig. 5). Unfortunately, the patient's melanoma progressed and he was awaiting further chemotherapy with Pembroluzimab.

\section{Discussion and conclusion}

MEK retinopathy usually presents acutely within the first week of the first dose. The retinal features described in MEK retinopathy include central serous retinopathy, serous retinal detachment, cystoid macular edema, intra-retinal fluid and cysts and thin choroid. Most of these features are identified on optical coherence tomography scans [6-10]. The retinopathy is typically bilateral and symmetrical [5-9]. In cases where only one eye is affected, other diagnoses should be considered [5]. Symptoms of MEK retinopathy can vary from being asymptomatic to blurred vision, altered color perception, shadows, light sensitivity, metamorphopsia and glare. Cases are often mild, short-lived, self-limiting, and do not interfere with activities of daily living $[8,11-$ 13]. Central retinal thickness and volume showed dose-dependent increases after the start of treatment, followed by a marked decrease despite continued treatment [8]. The retinopathy partially recovers, but can still be detected many months later [12]. Retinal thinning and retinal atrophy have been observed after long-term treatment [14] Cessation of life-extending treatment with MEK inhibitors is not indicated when SRF is present [12]. 


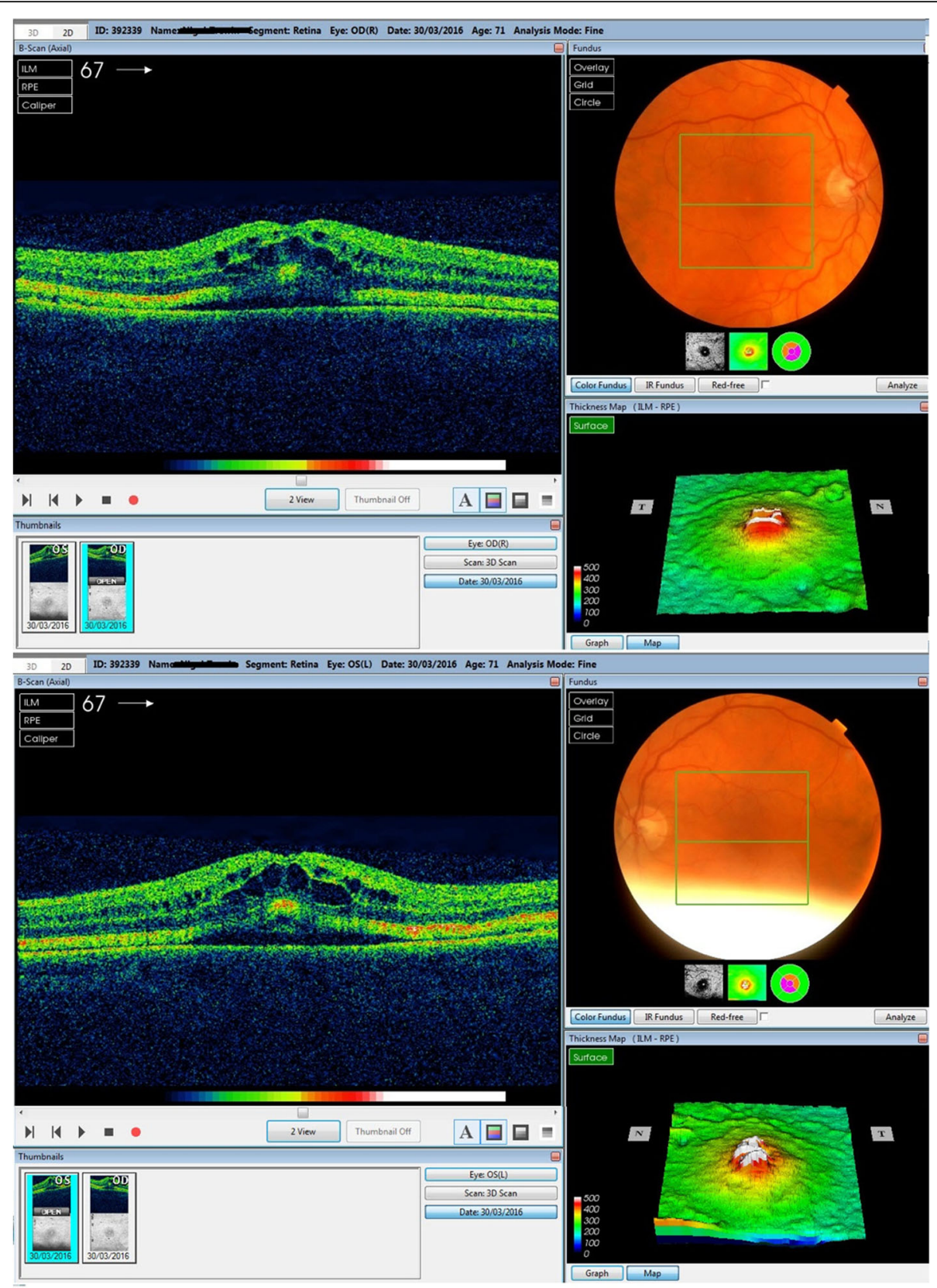

Fig. 1 OCT image at presentation: Bilateral cystoid macular edema at presentation

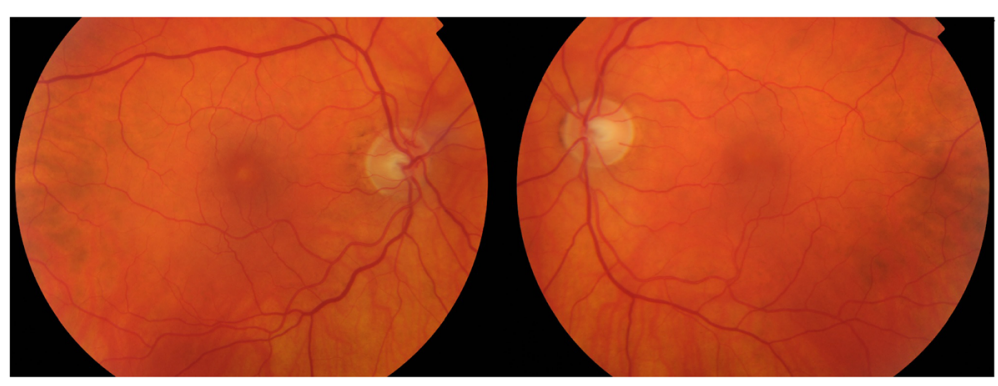

Fig. 2 Fundus appearance: Healthy optic disc, dull foveal reflex and normal vasculature 


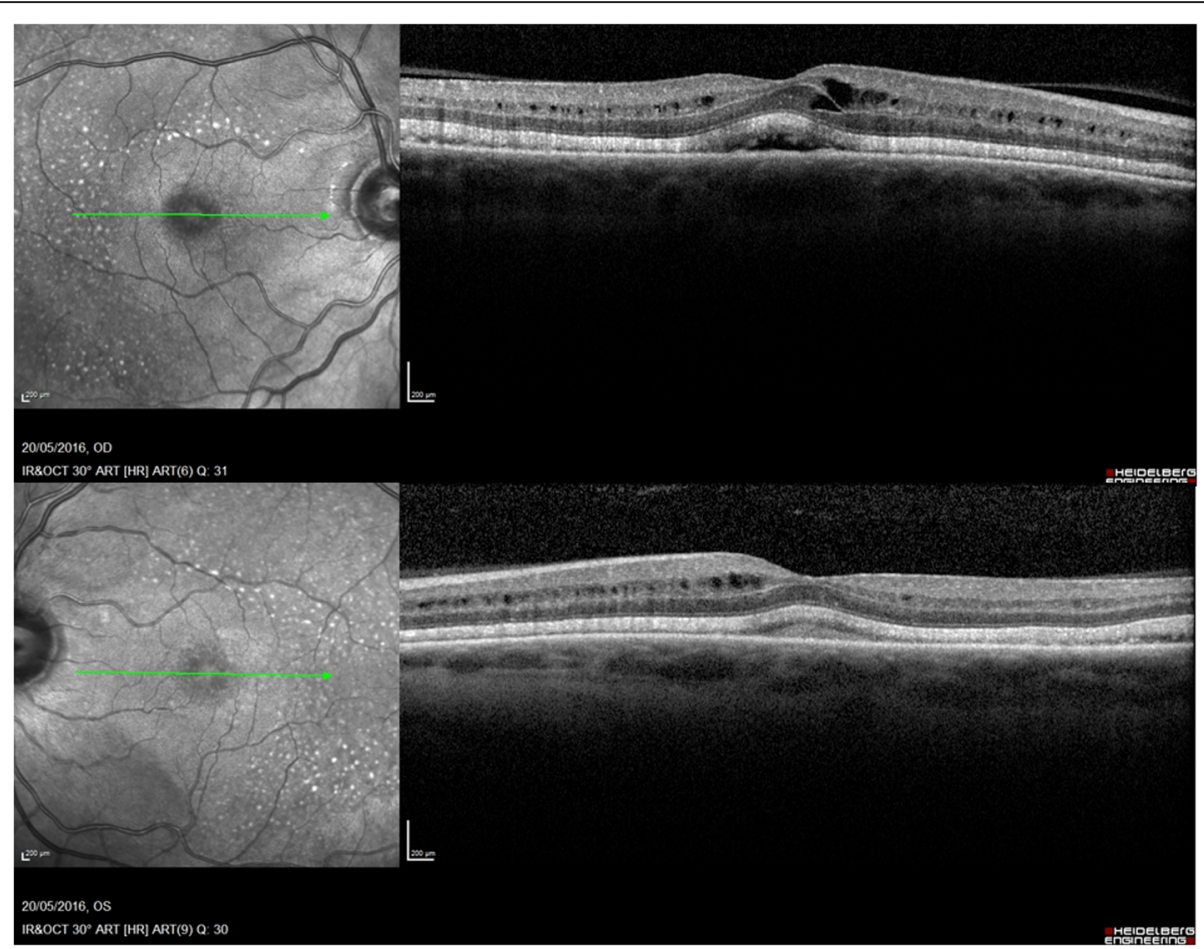

Fig. 3 OCT images 5 days after stopping treatment: Intra-retinal and sub-retinal fluid, thickening of ellipsoid zone, sub-retinal deposits and multiple hyper-fluorescent spots on IRR image

MEK pathway and its activation by the fibroblast growth factor receptor (FGFR) plays prominent role in the maintenance, survival and repair of RPE. Inhibition of this pathway leads to degeneration of RPE cells. The pathophysiology of MEK retinopathy is due to acute RPE toxicity which results in RPE hyperpermeability and breakdown of the retinal-blood barrier [15-17].

The treatment of MEK retinopathy is based on Common Terminology Criteria for Adverse Events (CTCAE) criteria, widely used for $\mathrm{AE}$ reporting in oncology studies, include a 4-category grading scheme for retinopathy according to symptom severity [18]. Asymptomatic patients and mild retinopathy with vision better than $6 / 12$ do not require interruption of dosing as mild symptoms and OCT abnormalities frequently resolve within days after continued dosing. This suggests that many patients with MEK retinopathy develop tachyphylaxis to continued MEK inhibitor therapy. For significant visual symptoms or vision below 6/ 12, patients should be instructed to interrupt dosing with MEK inhibitor therapy. When symptoms resolve, patients may be rechallenged at the same dose of MEK inhibitor therapy with close monitoring following re-initiation of treatment. For toxicities with severe visual impairment or interruption of daily activities, MEK inhibitor treatment

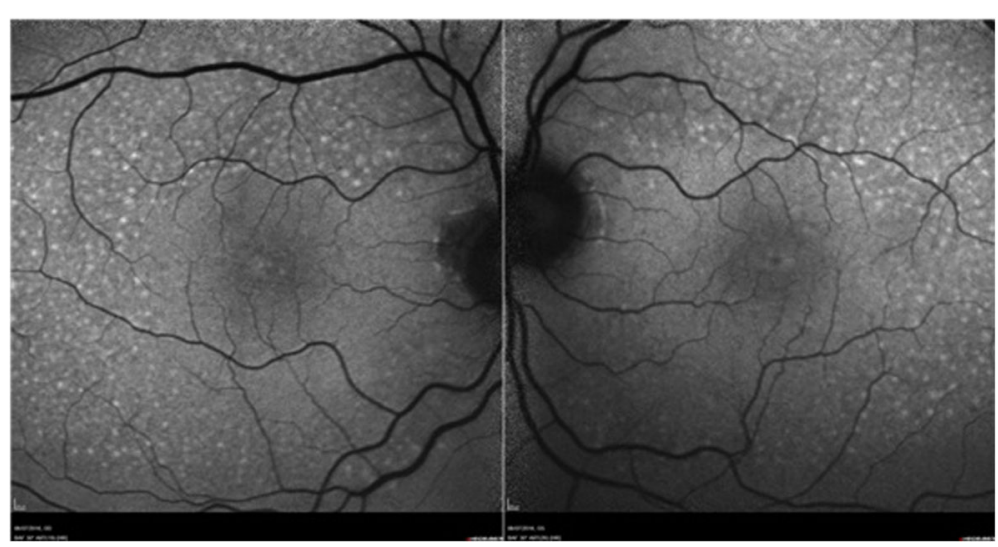

Fig. 4 Autofluorescence image: Increased autofluorescence of scattered sub-retinal granular deposits in macular area 


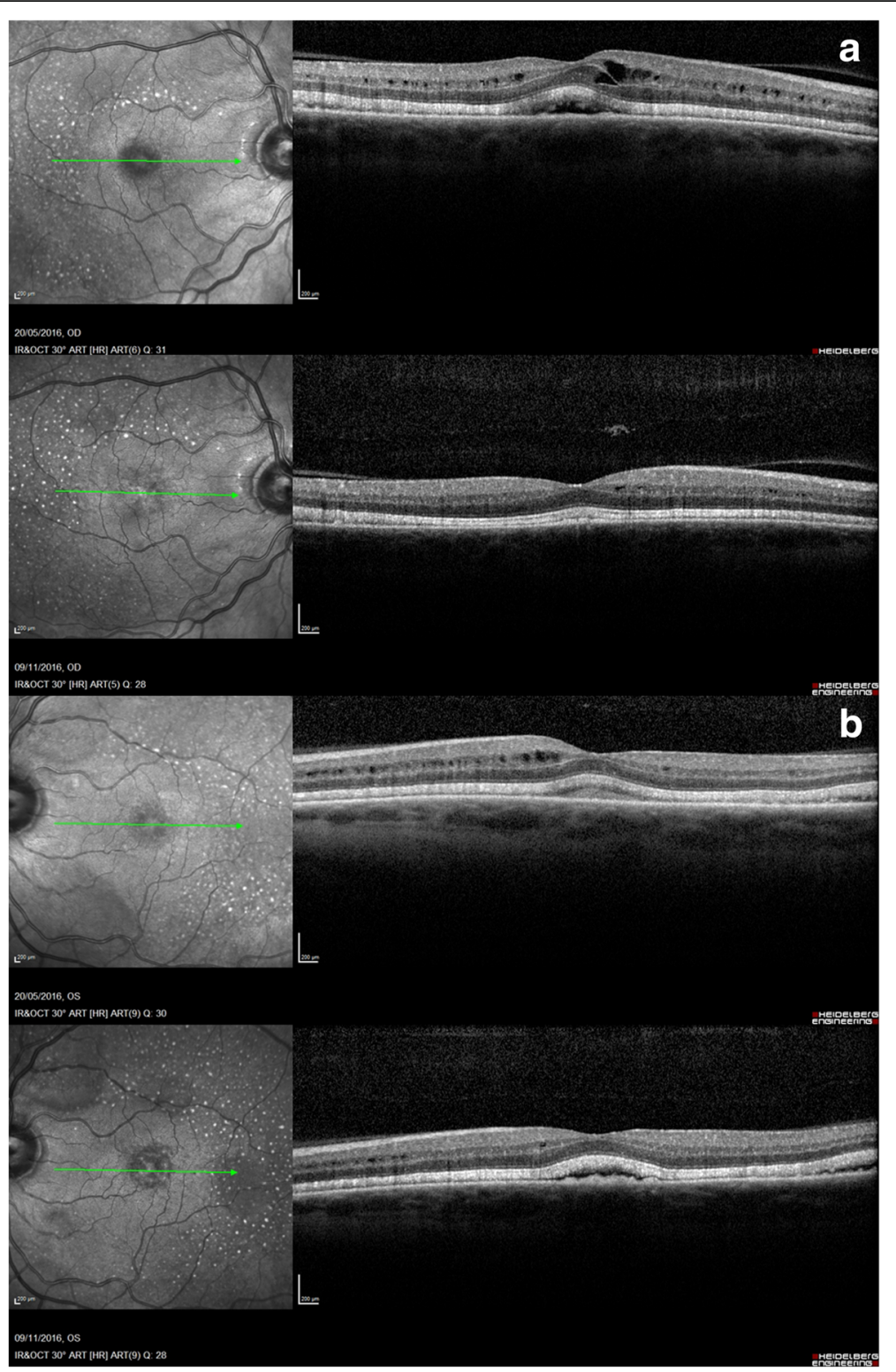

Fig. 5 Change in OCT appearance in both eyes over 6 months: Resolution of cystoid macular edema in perifoveal region, reduction in the intraretinal and sub-retinal fluid. The ellipsoid zone thickening and subretinal granular deposits remained unchanged in right (a) and left (b) eye

should be discontinued and when symptoms and OCT findings resolve, patients may be rechallenged at a lower dose $[5,18]$.

Our case showed bilateral, symmetrical changes within the retina in the form of cystoid edema, intra-retinal fluid and sub-retinal fluid signifying abnormal RPE permeability. Two new features were identified as thickening of ellipsoid zone and characteristically distributed subretinal granular deposits. The thickening of the ellipsoid zone could be due to swelling of the photoreceptors secondary to RPE toxicity and dysfunction affecting the photoreceptor nutrition. The subretinal granular deposits overlying a normal appearing RPE showed increased autofluorescence suggested abnormal lipofuscin clearance due to RPE dysfunction. Although the intra-retinal and sub-retinal fluid reduced, the ellipsoid layer changes and the granular changes persisted signifying ongoing RPE dysfunction despite reduced dosage even though the patient became asymptomatic. We were unable to perform fundus fluorescein angiography and electrodiagnostic tests which could have potentially shed more light on the structural and functional changes in retina.

In this case report we identified two new features of MEK retinopathy not previously described in literature. Introduction of molecularly targeted therapy have revolutionized the cancer treatment and increased the 
survival rate. Most of these agents are associated with ocular toxicities. These agents are relatively new and recently approved by NICE for clinical use in cancer treatment. Awareness of ocular symptoms, side-effect profile of drugs, monitoring regime and liaison between oncologist and eye care professional with ocular imaging is key to early diagnosis and management of ocular adverse events.

\section{Abbreviations}

AE : adverse event; MAPK/ERK, MEK : National Institute of Clinical Excellance: NICE mitogen-activated protein kinase/ extracellular regulated kinase; BD : twice a day; OD : once a day; OCT : optical coherence tomography; RPE : retinal pigment epithelium; AF : autofluorescence; FGFR : fibroblast growth factor receptor; CTCAE : Common Terminology Criteria for Adverse Events

\section{Acknowledgements}

We would like to acknowledge medical photographers, Mr. Victor Beattie, Mr. Tony Johnston and Mr. Martin Mcleod for taking clinical images, OCT and AF scans.

\section{Funding}

This supplement and the meeting on which it was based were sponsored by Novartis (tracking number OPT17-C041). Novartis did not contribute to the content and all authors retained final control of the content and editorial decisions. Novartis have checked that the content was compliant with the Association of the British Pharmaceutical Industry Code of Practice.

\section{Availability of data and materials}

Not applicable.

\section{About this supplement}

This article has been published as part of BMC Ophthalmology Volume 18 Supplement 1, 2018: The Novartis Ophthalmology Case Awards 2017. The full contents of the supplement are available online at https:// bmcophthalmol.biomedcentral.com/articles/supplements/volume-18supplement-1.

\section{Authors' contributions}

Both authors have read and approved manuscript.

Ethics approval and consent to participate

Not applicable.

\section{Consent for publication}

Written consent obtained from person reported in case presentation.

\section{Competing interests}

The authors declare that they have no competing interests.

\section{Publisher's Note}

Springer Nature remains neutral with regard to jurisdictional claims in published maps and institutional affiliations.

Published: 14 September 2018

\section{References}

1. Long GV, Menzies AM, Nagrial AM, et al. Prognostic and clinicopathologic associations of oncogenic BRAF in metastatic melanoma. J Clin Oncol. 2011; 29:1239-46.

2. Flaherty KT, Infante JR, Daud A, Gonzalez R, Kefford RF, Sosman J, Hamid O, Schuchter L, Cebon J, Ibrahim N, Kudchadkar R, Burris HA 3rd, Falchook G, Algazi A, Lewis K, Long GV, Puzanov I, Lebowitz P, Singh A, Little S, Sun P, Allred A, Ouellet D, Kim KB, Patel K, Weber J.Combined BRAF and MEK Inhibition in Melanoma with BRAF V600 Mutations. N Engl J Med. 2012; 367(18):1694-703.
3. Luke JJ, Ott PA. New developments in the treatment of metastatic melanoma - role of dabrafenib-trametinib combination therapy. Drug Healthc Patient Saf. 2014;6:77-88.

4. Menzies AM, Long GV. Dabrafenib and trametinib, alone and in combination for BRAF-mutant metastatic melanoma. Clin Cancer Res. 2014; 20:2035-43.

5. Stjepanovic N, Velazquez-Martin JP, Bedard PL. Ocular toxicities of MEK inhibitors and other targeted therapies. Ann Oncol. 2016;27:998-1005.

6. Velez-Montoya R, Olson J, Petrash M, et al. Acute onset central serous retinopathy in association with Mek inhibitor use for metastatic cancer. Invest Ophthalmol Vis Sci. 2011:52:21153.

7. van der Noll R, Leijen S, Neuteboom GH, et al. Effect of inhibition of the FGFR- MAPK signaling pathway on the development of ocular toxicities. Cancer Treat Rev. 2013;39:664-72.

8. Urner-Bloch U, Urner M, Stieger $P$, et al. Transient MEK inhibitor-associated retinopathy in metastatic melanoma. Ann Oncol. 2014;25:1437-41.

9. Schoenberger SD, Kim SJ. Bilateral multifocal central serous-like chorioretinopathy due to MEK inhibition for metastatic cutaneous melanoma. Case Rep Ophthalmol Med. 2013;2013:673796.

10. Duncan KE, Chang LY, Patronas M. MEK inhibitors: a new class of chemotherapeutic agents with ocular toxicity. Eye. 2015;29(8):1003-12.

11. van Dijk EH, van Herpen CM, Marinkovic M, Haanen JB, Amundson D, Luyten GP, Jager MJ, Kapiteijn EH, Keunen JE, Adamus G, Boon CJ. Serous retinopathy. Associated with mitogen-activated protein kinase kinase inhibition (Binimetinib) for metastatic cutaneous and uveal melanoma. Ophthalmology. 2015 Sep;122(9):1907-16.

12. Weber ML, Liang MC, Flaherty KT, Heier JS. Subretinal fluid associated with MEK inhibitor use in the treatment of systemic Cancer. JAMA Ophthalmol. 2016 Aug 1;134(8):855-62.

13. Niro A, Strippoli S, Alessio G, Sborgia L, Recchimurzo N, Guida M. Ocular. Toxicity in metastatic melanoma patients treated with mitogen-activated protein kinase kinase inhibitors: a case series. Am J Ophthalmol. 2015 Nov; 160(5):959-67.

14. Urner-Bloch U, Urner M, Jaberg-Bentele N, Frauchiger AL, Dummer R, Goldinger SM. MEK inhibitor-associated retinopathy (MEKAR) in metastatic melanoma: long-term ophthalmic effects. Eur J Cancer. 2016 Sep;65:130-8.

15. Jiang $\mathrm{Q}$, Cao C, Lu S, et al. MEK/ERK pathway mediates UVB-induced AQP downregulation and water permeability impairment in human retinal pigment epithelial cells. Int J Mol Med. 2009;23:771-7.

16. Kuznetsova AV, Kurinov AM, Aleksandrova MA. Cell models to study regulation of cell transformation in pathologies of retinal pigment epithelium. J Ophthalmol. 2014;2014:801787.

17. Huang W, Yang AH, Matsumoto D, et al. PD0325901, a mitogen-activated protein kinase kinase inhibitor, produces ocular toxicity in a rabbit animal model of retinal vein occlusion. J Ocul Pharmacol Ther. 2009;25:519-30.

18. National_Cancer_Institute. Common Terminology Criteria for Adverse Events v4.0. NCl, NIH, DHHS. NIH publication \# 09-7473, 29 May 2009.

\section{Ready to submit your research? Choose BMC and benefit from:}

- fast, convenient online submission

- thorough peer review by experienced researchers in your field

- rapid publication on acceptance

- support for research data, including large and complex data types

- gold Open Access which fosters wider collaboration and increased citations

- maximum visibility for your research: over $100 \mathrm{M}$ website views per year

At BMC, research is always in progress.

Learn more biomedcentral.com/submissions 